\title{
Sistema integrado para toma de decisiones en el diseño de estructuras de hormigón
}

\author{
An integrated decision-making methodology for the \\ design of concrete structures
}

L. Armengou $^{(*)}$, A. Aguado ${ }^{(* *)}$, G. Ormazábal ${ }^{(* * *)}$

\section{RESUMEN}

El presente artículo hace una breve reflexión sobre las alternativas in situ y prefabricadas para estructuras de hormigón, justificando la necesidad de profundizar en métodos multicriterio como ayuda a la toma de decisiones. Manifiesta la trascendencia de la calidad en las decisiones más allá de este ámbito concreto, así como su influencia en la mayor eficiencia de las obras. Presenta luego un sistema de ayuda a la toma de decisiones basado en matemática difusa, que considera el valor de cada alternativa contemplando el riesgo inherente. Para ilustrarlo desarrolla un ejemplo aplicado a pasos inferiores que pueden resolverse mediante prefabricación o in situ. Efectuándolo por completo, llega a dos conclusiones principales: la bondad de la herramienta presentada y cómo la solución prefabricada, en este caso, aporta mayor valor. Concluye también que todo buen gestor debe plantearse su toma de decisión desde los inicios de cualquier proyecto, empleando herramientas como la presentada.

450-25

Palabras clave: Análisis multicriterio; toma de decisiones; IDS; prefabricados de hormigón; hormigón in situ.

\section{SUMMARY}

This paper presents a methodology to analyze decisions in the design of concrete structures. Specifically, we propose a multi-criteria approach to assess the value implications of precast concrete technologies versus in-situ concrete solutions. First, we emphasize the importance of careful decision-making in early stages of construction projects to enhance customer value. Second, we present a decisionmaking methodology that uses fuzzy logic to represent and synthesize information about risk-return tradeoffs in the different kinds of variables involved in the evaluation of a project's value. To illustrate, we use our methodology to analyze precast vs. in-situ solutions in the design of drainage structures. The results of our analysis point at precast solutions as the most desirable design choice in this setting, and highlight the importance of value analysis in early stages of construction projects.

Keywords: Multi-criteria analysis; decision making; IDS; precast concrete; "in situ" concrete.

\footnotetext{
(*) ETSA-Universitat Internacional de Catalunya. Barcelona (España).

(**) Universitat Politècnica de Catalunya. Barcelona (España).

(***) IESE Business School, Universidad de Navarra. Barcelona (España). 


\section{INTRODUCCIÓN}

Las soluciones prefabricadas, tanto en obra civil como en edificación, que sustituyen a soluciones usualmente resueltas en hormigón in situ es cada vez mayor (1) (2), siendo antiguo el debate de las ventajas e inconvenientes de ambos tipos de solución y su presencia en diferentes mercados (3) (4) (5).

Una forma de cuantificar las características de cada alternativa, para la toma de decisiones en la gestión de proyectos, es mediante técnicas multicriterio. Estas empezaron a tratarse de forma teórica en el ámbito de la economía y la gestión empresarial'

Sin embargo, a lo largo de los años empezó a despertarse el interés por la cuestión en el ámbito de la construcción, hasta el reciente desarrollo de diversos modelos para la toma de decisión en diversos aspectos de la gestión de la misma, principalmente en torno a la contratación, la construcción y el diseño, estudio de alternativas, y la resolución de conflictos ${ }^{2}$.

Actualmente, la trascendencia de la toma de decisión en la gestión de proyectos constructivos es algo universalmente reconocido, tanto en el mundo profesional como en el ámbito académico ${ }^{3}$. En este sentido, Aibinu (25) correlaciona la eficiencia de trabajos de construcción con la calidad de las tomas de decisión; la calidad requiere, según dicho autor, neutralidad, precisión y consistencia. Se necesita el apoyo en hechos y no en opiniones para decidir con fundamento; sin embargo, la evaluación de acciones que todavía no han ocurrido requiere el uso de metodologías que disminuyan el efecto de la subjetividad de los agentes en la pérdida de calidad del proceso de toma de decisiones.

En el marco de la prefabricación, el problema de la toma de decisión adquiere especial relevancia al plantearse el frecuente dilema entre la ejecución in situ y una posible solución prefabricada. La cuestión no es trivial, ya que en ella inciden aspectos no sólo de carácter técnico, sino que la creciente complejidad de la demanda del sector de la construcción, motivada por los recientes cambios que ha experimentado nuestra sociedad, hacen que intervengan cada vez más parámetros en la toma de decisión (32). A las cada vez más exigentes variables tradicionales: el coste, el plazo y la calidad, se añaden nuevos requisitos como la seguridad de los operarios, el respeto al medioambiente o el ahorro de recursos naturales.

Es en este marco donde se propone la aplicación de un método de toma de decisiones denominado «Integrated Decision Sys- tem» (33) en la elección entre una solución in situ y otra prefabricada. El citado método aporta un modelo o estructura de razonamiento para el o los responsables de tomar la decisión que integra los diversos aspectos anteriormente mencionados.

Asimismo, incorpora también un aparato matemático que a través de la lógica difusa ('fuzzy logic') permite una evaluación flexible de las diversas alternativas, teniendo en cuenta que algunos de los parámetros serán de carácter cuantitativo (costes, plazos, variables físicas) y otros tendrán un cariz más intangible (aspectos estéticos, sociales, etc.). Por consiguiente, el objetivo del sistema de toma de decisiones propuesto es servir de apoyo metodológico para conseguir un estudio más riguroso de la decisión, objetivándola al máximo e integrando de forma coherente las diversas vertientes y las múltiples variables del problema, las cuales dependerán de las características del caso considerado.

Podríamos, sin duda, acudir a otras metodologías, dentro de la misma lógica difusa, o de tipo análisis multicriterio (34); sin embargo, aparte de cuál es el método que describamos aquí, es relevante el hecho de que el proceso de análisis ya obliga a una búsqueda del planteamiento de alternativas y del consenso que serán un paso imprescindible para lograr la idoneidad de la solución escogida y facilitar su implantación sin conflictos ni ineficiencias.

El empleo de la lógica o matemática difusa ('fuzzy') como mejora sobre otras metodologías se puede fundamentar en su capacidad de modelizar la ambigüedad (35) (36). Mills (37), ya dentro del ámbito de la ética, habla de cómo la estructura de valores repercute en la toma de decisiones. Sin embargo, el paso de una estructura de valores a una decisión concreta es justamente lo que requiere un análisis de alternativas neutro, preciso y consistente.

Este artículo tiene por objeto presentar el modelo multicriterio IDS y mostrar un ejemplo de aplicación para la construcción de pasos inferiores, analizando soluciones in situ y prefabricadas.

\section{SOLUCIÓN PREFABRICADA O IN SITU: CUANDO SÓLO UNA ALTERNATIVA ES ÓPTIMA}

El sector de la construcción es uno de los más relevantes en la economía mundial y de mayor repercusión en la calidad de vida de las personas. La rápida evolución del conocimiento y su puesta en común entre la comunidad profesional y académica mundial ha extendido dos ámbitos de modo particular: 
- Las metodologías de apoyo a la toma de decisiones en la gestión de proyectos, desde las grandes a las pequeñas decisiones.

- Las posibilidades de los sistemas prefabricados para dar soluciones de alta eficiencia en la confección de estructuras desde puntos de vista muy diversos (económico, temporal, funcional, social y medioambiental)

Aunque hay evidencias de la superioridad de la solución prefabricada para muchos casos, no siempre las decisiones tomadas son coherentes con lo recogido hasta aquí. A manera de ejemplo, la Figura 1 presenta las satisfacciones medias bajo diversos puntos de vista de soluciones prefabricadas e in situ en Hong Kong para edificios residenciales y no residenciales (4), si bien esto no quiere decir que la alternativa prefabricada sea siempre la solución óptima.

Sacks (3), Jaillon (4), Polat (5), entre muchos autores, nos descubren la desigual presencia de las soluciones prefabricadas en sociedades de las que podríamos esperar una respuesta similar. Datos de 1998 recogidos por Polat (5) indican extremos sólo parcialmente explicables: $56 \%$ como ratio prefabricado $v s$ total en Finlandia frente a un $6 \%$ en EEUU. Alemania y el Reino Unido están entre el 25 y el $30 \%$, mientras que la situación en España, puede darse como valor medio un $20 \%$, si bien depende, lógicamente, del tipo de aplicación.

Una razón para explicar esas diferencias, puede buscarse en condiciones climáticas; evidentemente que en países muy fríos la prefabricación será una solución ineludible para muchos casos, pero eso no justifica tales diferencias. Otras razones pueden ser culturales de países o de otro tipo (38).

Polat (5) intenta entender la razón de ser que el uso del prefabricado en EEUU no sea mayor. Para ello elimina interferencias, tales como:

- No hay dificultades relevantes en la incompatibilidad de los productos por falta de estandarización, si es que eso puede ser una dificultad.

- Las cuestiones estéticas se satisfacen mediante el diseño.

- Los usuarios de edificios prefabricados manifiestan su satisfacción de modo inequívoco.

- El comportamiento sísmico de las estructuras prefabricadas no es diferente del de las estructuras resueltas con hormigón in situ.

En ese contexto, considera más adelante que un factor principal en el bajo uso del prefabricado allá donde sería adecuado es

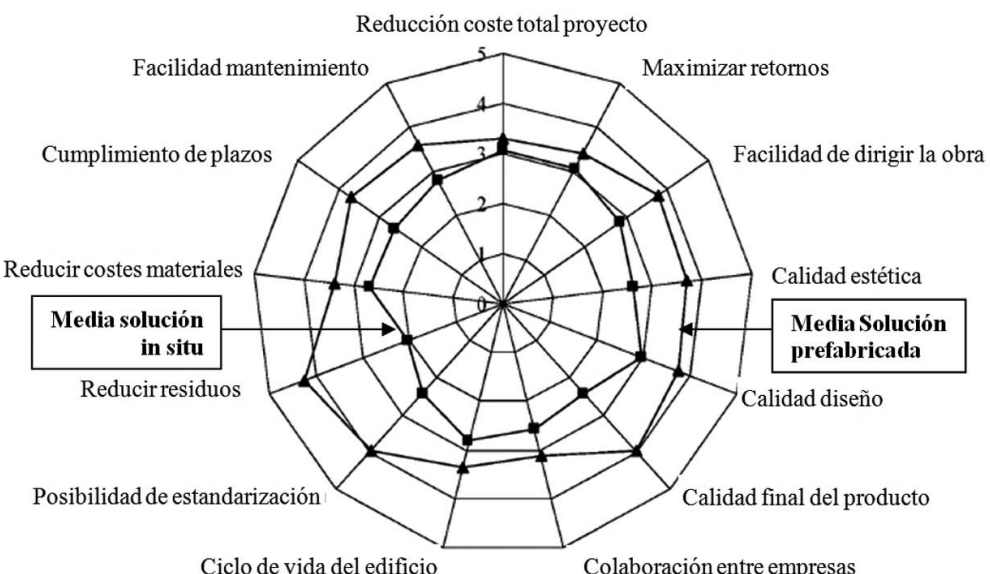

la falta de profesionales que puedan diseñar y gobernar la construcción con elementos prefabricados. Incluso se puede pensar en la repercusión negativa que puedan haber tenido errores de diseño o concepción en la cultura colectiva.

Otro aspecto a considerar es cuál debe ser el momento en que se opta por la solución prefabricada. Si un proyecto se redacta pensando en la solución in situ, el cambio durante la fase de construcción a prefabricado estará muy condicionado; por tanto, el gestor del proyecto tiene que tomar sus decisiones en las fases más tempranas del diseño.

La falta de profesionales bien formados, no obstante, no anula la responsabilidad de los ingenieros y arquitectos presentes en cualquier mercado: el conocimiento está disponible y la actualización profesional es un deber inherente de cualquier trabajador. Todo ello pone en evidencia la necesidad de formar a los estudiantes de ingeniería y arquitectura en el uso de las tecnologías de la prefabricación.

Para reducir los riesgos de equivocarse en la toma de decisiones, y pensando en los nuevos profesionales, parece razonable pensar en incrementar el papel de los métodos de análisis multicriterio. En este contexto se sitúa el sistema integrado de decisiones (IDS) que se presenta en este artículo.

\section{LA HERRAMIENTA DE ANÁLISIS: EL SISTEMA IDS}

El sistema IDS propuesto por Ormazábal (33) se estructura en tres elementos principales: el concepto de valor y su modo de medición o evaluación, el concepto de riesgo y su tratamiento matemático y un proceso metodológico denominado ACE con relación a sus fases principales (Análisis, Creatividad y Evaluación), donde se integran los dos elementos anteriormente especificados. En lo que sigue se hace una breve revisión de estos elementos. 


\subsection{El valor. Concepto y medición}

En la toma de una cierta decisión en el marco del desarrollo de un proyecto de construcción, el valor de una alternativa relativa se define como el grado de satisfacción que produce, medido como la respuesta a los requerimientos del proyecto, considerando diversos planos: económico (\$), temporal $(\mathrm{T})$, funcional $(\mathrm{F})$, social $(\mathrm{S})$ y medioambiental (M). Los requerimientos se pueden agrupar de otras formas o bien ser diferentes a los señalados. Por otro lado, cabe señalar que el concepto de valor utilizado en este trabajo no debe confundirse con la estructura de valores de las teorías éticas.

La medición del valor se articula a través de una función, $v(x)$, definida entre 1 y -1 que representa el grado de satisfacción que produce un parámetro de respuesta respecto a un cierto requerimiento ( 1 máxima satisfacción y -1, mínima satisfacción) También sería posible representar la satisfacción de 0 a 1, tal como se hace en la metodología MIVES (39).

Las funciones de valor de los distintos requerimientos se integran mediante un árbol de requerimientos, donde se pondera la importancia relativa de cada uno de ellos y sus relaciones correspondientes. El despliegue de dicho árbol dará pie al cálculo del valor global de la alternativa, medido mediante las expresiones [1] y [2]. donde

$V=\sum_{i_{1}=1}^{n_{1}} k_{i_{1}}^{1} \cdot \sum_{i_{2}=1}^{n_{2}} k_{i_{2}}^{2} \cdot \cdots \cdot \sum_{i_{m}=1}^{n_{m}} k_{i_{m}}^{m} \cdot v_{i_{m}}^{m}\left(x_{1}^{i_{m}}, x_{2}^{i_{m}}, \ldots, x_{l}^{i_{m}}\right)$

siendo:

$$
v_{i_{m}}^{m}\left(x_{1}^{i_{m}}, x_{2}^{i_{m}}, \ldots, x_{l}^{i_{m}}\right)=\sum_{k=1} w_{k}^{i_{m}} \cdot v_{i_{m}}^{m}\left(x_{k}^{i_{m}}\right)
$$

$v_{j}^{i}(x)$ las funciones de valor de los diversos requerimientos, $x_{j}^{i}$ los parámetros de respuesta, $k_{j}^{i}$ el peso relativo de cada [sub] requerimiento según el árbol definido y $w_{j}^{i}$ la importancia relativa de los diversos parámetros que intervienen en la evaluación de un cierto requerimiento. La ponderación de los pesos se realizará aplicando el método AHP (Analytical Hierarchy Process) de Saaty (40), a través de una comparación por pares de los mismos.

\subsection{El riesgo. Concepto y tratamiento matemático}

El concepto de riesgo se define como una incertidumbre en el juicio por la falta de conocimiento perfecto en la previsión de los resultados relativos a los diversos planos de estudios considerados o por la posible variación de estos a causa de sucesos o agentes que tengan influencia sobre la realidad considerada. Se distingue, por tanto, entre dos tipos de riesgo, con un tratamiento matemático diferenciado:

- riesgos especulativos: se define así la incertidumbre asociada al conocimiento de un cierto resultado.

- riesgos puros: son los factores que pueden producir una variación en el valor del proyecto, produciendo una modificación en los parámetros de respuesta (sobrecostes, retrasos, etc.).

El tratamiento de los riesgos especulativos se articula a través del uso de la matemática difusa ('fuzzy logic'), usualmente aplicada en el entorno de las técnicas de inteligencia artificial. En concreto, se diseñan un elemento al que se le denomina "trapecio difuso" (Figura 2) y que constituye la base del planteamiento (41). Según se observa en la citada Figura 2, dicho trapecio modeliza la incertidumbre asociada al conocimiento de un cierto parámetro (por ejemplo el coste o el tiempo de ejecución de una determinada unidad de obra). En él se representa un intervalo más probable (b-c), y dos intervalos donde es menos probable que se encuadre el valor numérico del parámetro $(a-b$ y c-d). El grado de probabilidad se modeliza mediante la función de pertenencia, $\mu(x)$, usual en el ámbito de la matemática difusa.

La ventaja principal de este nuevo planteamiento es que evita el uso de la probabilidad, lo cual es especialmente importante en un sector como la construcción, donde los productos son singulares y no existe la repetibilidad necesaria para plantear la evaluación de probabilidades.

Por otro lado, la evaluación de los riesgos puros se articula mediante una función de severidad, definida como la pérdida de valor que produce el factor de riesgo respecto al requerimiento considerado. Su medición se define a partir de la función de valor inicialmente planteada, según las expresiones [3] y [4], visualizada en la Figura 3.

[3]

$$
s\left(y, x_{0}\right)=\left|v\left(x_{0}\right)-v(y)\right|
$$

donde $y$ es el valor del parámetro $x$ tras el impacto del factor de riesgo considerado, es decir,

$$
y=x_{0}+\Delta x
$$

A partir de las funciones de severidad relativas a cada uno de los requerimientos a los que afecta el riesgo puro considerado, se calculará una severidad total del modo análogo al indicado en las expresiones [1] y [2], a través de la media ponderada según la estructura y pesos del árbol de requerimientos. Dicha severidad deberá substraerse del valor inicial, teniendo en cuenta los 

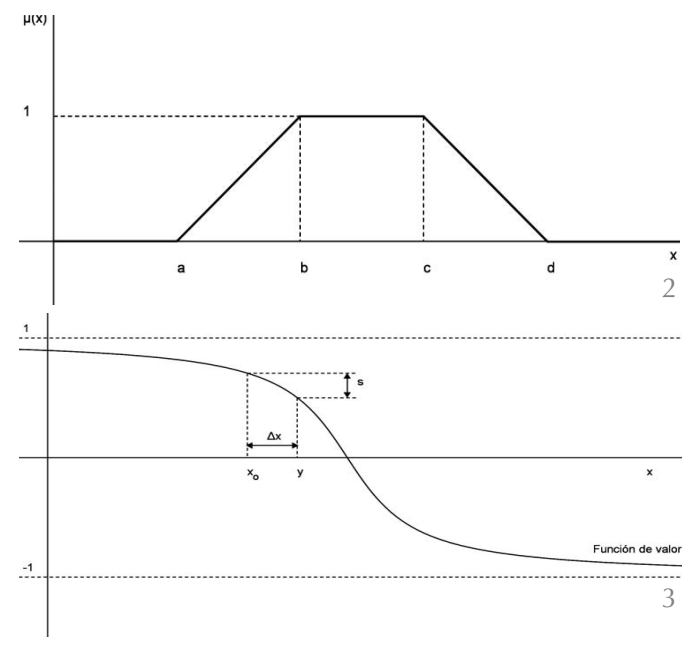

3

Tabla 1. Esquema del proceso ACE (33)

\begin{tabular}{|c|c|c|c|c|c|c|}
\hline Fase & $\begin{array}{c}\text { Nomen- } \\
\text { clatura }\end{array}$ & \multicolumn{5}{|c|}{ Etapas } \\
\hline \multirow{5}{*}{ Análisis } & A1 & \multicolumn{5}{|c|}{ Preparación del estudio y recopilación de información } \\
\hline & A2 & \multicolumn{5}{|c|}{ Identificación de procesos; construcción de la matriz de procesos } \\
\hline & A3 & \multicolumn{5}{|c|}{$\begin{array}{l}\text { Identificación de requerimientos; construcción de la matriz } \\
\text { de requerimientos }\end{array}$} \\
\hline & A4 & \multicolumn{5}{|c|}{ Construcción del árbol de requerimientos } \\
\hline & A5 & \multicolumn{5}{|c|}{$\begin{array}{l}\text { Análisis comparativo de los requerimientos; ponderación } \\
\text { y asignación de pesos }\end{array}$} \\
\hline \multirow{2}{*}{ Creatividad } & $\mathrm{C} 1$ & \multicolumn{5}{|c|}{ Identificación de puntos de decisión y definición de su alcance } \\
\hline & $\mathrm{C} 2$ & \multicolumn{5}{|c|}{ Generación de alternativas para cada punto de decisión } \\
\hline \multirow{8}{*}{ Evaluación } & E1 & \multicolumn{5}{|c|}{ Preselección cualitativa de alternativas en cada punto de decisión } \\
\hline & \multirow{7}{*}{ E2 } & \multirow{7}{*}{ 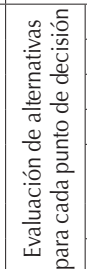 } & E2.1 & \multicolumn{3}{|c|}{ Evaluación del valor sin riesgos } \\
\hline & & & E2.2 & \multicolumn{3}{|c|}{ Análisis y evaluación de los riesgos de la toma de decisión } \\
\hline & & & E2.3 & \multicolumn{3}{|c|}{ Cálculo del valor integrado } \\
\hline & & & E2.4 & \multicolumn{3}{|c|}{ Análisis de compatibilidad } \\
\hline & & & \multirow[t]{2}{*}{ E2.5 } & \multirow{2}{*}{$\begin{array}{l}\text { Análisis } \\
\text { de los resultados }\end{array}$} & E2.5.1 & $\begin{array}{l}\text { Cálculo del índice de } \\
\text { potencialidad y eficiencia }\end{array}$ \\
\hline & & & & & $\mathrm{E} 2.5 .2$ & Análisis de sensibilidad \\
\hline & & & E2.6 & \multicolumn{3}{|c|}{ Toma de decisión y justificación; presentación de resultados } \\
\hline
\end{tabular}

diversos riesgos puros que intervienen, lo cual se expresa matemáticamente de modo general en la expresión [5].

$$
v=v^{*}-s
$$

donde:

$v^{*}$ es el valor calculado sin tener en cuenta la influencia de los riesgos puros y $s$ es la severidad esperada considerando los distintos riesgos que intervienen $\left(R_{l}, \ldots, R_{n}\right)$, es decir,

[6]

$$
\begin{gathered}
s=p_{R_{1}} \cdot s_{R_{1}}+\cdots+p_{R_{n}} \cdot s_{R_{n}}+p_{R_{1} \cap R_{2}} \cdot \\
\cdot s_{R_{1} \cap R_{2}}+\cdots+p_{R_{1} \cap R_{2} \cap \ldots \cap R_{n}} \cdot s_{R_{1} \cap R_{2} \cap \ldots \cap R_{n}}
\end{gathered}
$$

\subsection{El proceso ACE de toma de decisión}

Los conceptos y modos de evaluación definidos anteriormente se integran en un proceso metodológico denominado ACE, cuya estructura es la indicada en la Tabla 1. Hay que llamar la atención de que dicho proceso se define de forma flexible, de modo que permite una retroalimentación continua entre sus fases, al final de las mismas, de forma tal que la salida de una fase pueda servir de retorno para entrar en y corregir en una fase previa.

Por otro lado, dicho proceso se plantea como una estructura de razonamiento, aplicable de modo cuantitativo mediante el uso de los modos de medición anteriormente explicados, o de manera cualitativa, a través de la consideración de los modelos conceptuales y la estructura de trabajo que introduce.

En el caso del sector de la construcción, el proceso ACE puede venir prefijado previamente por las alternativas en estudio teniendo pocas opciones de a través de la etapa de Creatividad, volver a modificar las soluciones (alternativas). Sólo será viable en alguna tomas de decisiones específicas.

\section{CASO DE APLICACIÓN}

\subsection{Características de las alternativas}

Cara a concretar el planteamiento abstracto dado con anterioridad se presenta un caso de estudio, el cual se encuadra en las obras de una urbanización de la cala Calafató, en Atmetlla de Mar (Tarragona), a pocos metros de la costa, en un paraje de gran valor ecológico. En este caso, la propiedad de la obra correspondía a la promotora VERTIX, y el contratista fue la empresa COPCISA. En dicho proyecto se dispusieron tres obras de drenaje para permitir el flujo de pequeños torrentes hacia el mar por debajo de los viales. En este contexto, se plantea la alternativa de substituir la solución inicial del proyecto, prevista para ejecutar in situ, por una solución con clave prefabricada y hastiales in situ. Ambas alternativas se representan en la Figura 4 y Figura 5.

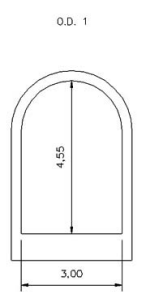

0.0 .1
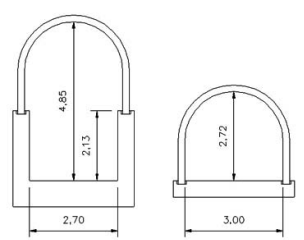

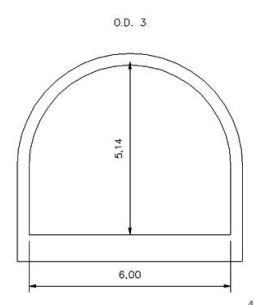

0.0 .3

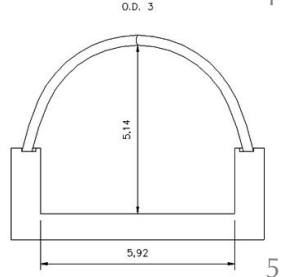

2. Trapecio difuso genérico.

3. Definición de la severidad a partir de la función de valor.

4. Solución in situ según el proyecto inicial.

5. Solución prefabricada

Como puede observarse en la Figura 5, la solución 'prefabricada' se compone realmente en este caso de unos hastiales in situ y una clave prefabricada. Ello era debido a la falta de disponibilidad de elementos prefabricados en el mercado. Los hastiales se ejecutarían in situ al no existir en el mercado moldes con tanta altura de hastial para 
6. Despliegue del árbol de valor para la evaluación de alternativas. (NDE: Nivel Deseado de Estudio).

la O.D. 1; al ser una obra pequeña ningún industrial está dispuesto a comprar un molde para tan poco metraje. Además, en caso de llevarlo a cabo redundaría en un aumento considerable de plazo. El diseño se modifica ligeramente respecto a la solución inicial por varios motivos. En primer lugar, para adaptar el diseño in situ a los moldes disponibles. Por otro lado, se busca unificar las secciones de la O.D. 1 y la O.D. 2 a la misma pieza prefabricada para bajar el precio unitario aumentando el número de piezas prefabricadas. Finalmente, se pretende adaptar la sección de la O.D. 3 a otra que, en ese momento, se estaba fabricando para otro cliente, lo cual permitía bajar el precio al dar continuidad a una producción ya iniciada.

Con el objetivo de discernir si la nueva solución prefabricada era más satisfactoria que la alternativa inicial in situ se planteó la aplicación del sistema IDS. Para ello se aplica el proceso ACE anteriormente descrito. El interés de este caso radica en que este tipo de substitución de bóvedas prefabricadas es muy común en las estructuras de carreteras, y en ocasiones suponen muchos metros lineales, y por tanto grandes sumas de dinero.

\subsection{Fase de Análisis}

La identificación de los requerimientos del proyecto se realiza a través del esquema

Tabla 2. Árbol de requerimientos del proyecto estudiado

\begin{tabular}{|l|l|c|}
\hline Requerimiento & \multicolumn{1}{|c|}{ Indicador } & Peso \\
\hline \multirow{4}{*}{ Económico } & Aumento de la actividad económica & 0,276 \\
\cline { 2 - 3 } & Costes de construcción & 0,063 \\
\cline { 2 - 3 } & Costes de uso & 0,080 \\
\hline \multirow{2}{*}{ Tiempo } & Plazo de ejecución & 0,018 \\
\cline { 2 - 3 } & Tiempo de trayecto & 0,016 \\
\hline \multirow{3}{*}{ Calidad } & Buen funcionamiento & 0,143 \\
\cline { 2 - 3 } & Estética de la obra & 0,052 \\
\cline { 2 - 3 } & Comodidad de uso & 0,080 \\
\hline \multirow{3}{*}{ Social } & Desarrollo del entorno social & 0,105 \\
\cline { 2 - 3 } & Seguridad e higiene en construcción & 0,011 \\
\cline { 2 - 3 } & Seguridad de los usuarios & 0,133 \\
\hline \multirow{2}{*}{ Medio ambiente } & Respeto al entorno durante construcción & 0,014 \\
\cline { 2 - 3 } & Respeto al medioambiente en uso & 0,009 \\
\hline
\end{tabular}

matricial considerando dos ejes: las diversas etapas del ciclo de vida del proyecto y los planos de análisis integrados en el concepto de valor propuesto, según se mostraba en la Tabla 1 (económico, temporal, funcional, social y medioambiental). A partir de esta identificación se construye el árbol de requerimientos, mostrado en la Tabla 2, y se lleva a cabo su ponderación relativa según el método descrito por Ormazábal (33).

Los requerimientos identificados hacen referencia al conjunto del proyecto, de modo que la decisión aquí considerada no afectará a muchos de ellos. Por otro lado, en la Figura 5 no se muestran otros requerimientos identificados por considerarlos de menor importancia en el conjunto del valor del proyecto. Como es obvio, en este caso huelga llevar a cabo la fase de creatividad del proceso ACE, pues el punto de decisión y las alternativas ya están fijados.

\subsection{Fase de evaluación}

Para el cálculo del valor sin considerar los riesgos puros de la toma de decisión, el director de obra despliega el árbol inicial de requerimientos identificando los parámetros de respuesta correspondientes según lo indicado en la Figura 6.

En la figura anterior puede observarse que los parámetros de medición de la respuesta de las alternativas Ilevan asociado un peso, correspondiente a su grado de participación en el conjunto de la satisfacción del [sub]requerimiento correspondiente.

Respecto al coste, debe considerarse, en primer lugar, que el prefabricado Ileva consigo la necesidad de transporte, que en algunos casos redunda de forma importante en el precio. En este caso, dado el tamaño de las piezas, no tiene una gran importancia. Sin embargo, el diseño del prefabricado es más optimizado al ejecutarse con un control más
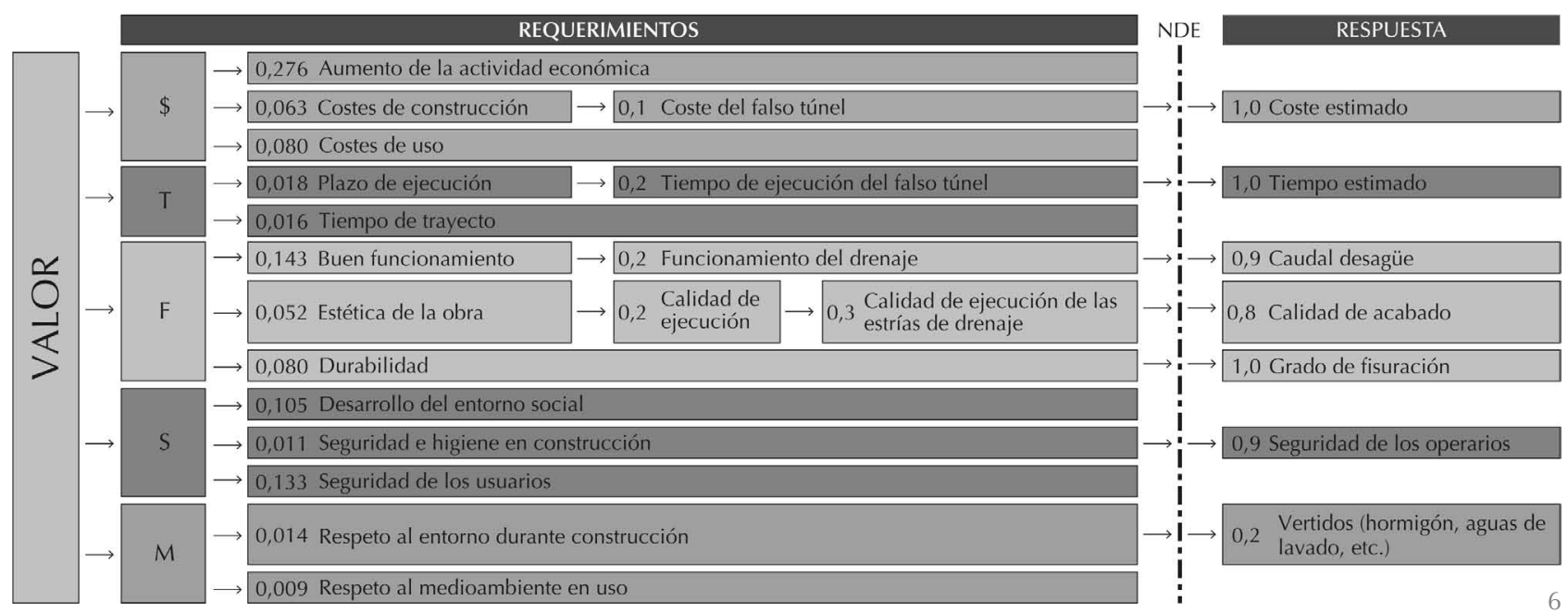
intenso. Este control no sólo mejora el diseño sino que además permite abaratar costes al disminuir factores de seguridad. De hecho, en este caso, la solución prefabricada supone un coste algo inferior a la inicialmente proyectada. Asimismo, la asistencia técnica del proveedor del prefabricado puede ahorrar horas de ingeniería a la constructora, además de aportarle una experiencia y asesoramiento técnico de gran valor.

En lo relativo al plazo, la prefabricación supone un claro ahorro de tiempo, pues evita los inconvenientes propios de la producción a pie de obra y los tiempos de vertido, vibrado, fraguado desencofrado y curado.

Desde un punto de vista funcional, la prefabricación supone en este caso tres claras ventajas. Por un lado, implica un acabado mejor, consecuencia de un trabajo en condiciones menos adversas. Además, supone una mayor durabilidad, la cual se asocia al nivel de fisuración (se impone el mismo límite al hormigón prefabricado e in situ) y a la $f_{c k}$. El prefabricado aportará ventajas en este sentido al conseguir resistencias mayores que las relativas a la ejecución in situ. Finalmente, el mejor acabado del producto prefabricado redunda en una menor rugosidad del conducto de drenaje, lo que implica una mayor capacidad de desaguar (por tener un coeficiente de Manning menor y por tanto un caudal de desagüe mayor). Obviamente, sólo ocurre en igualdad del resto de condiciones $\left(R_{h}, i\right.$, $S$, etc.) y es necesario tener en cuenta que si bien la rugosidad aumenta por las juntas - no olvidemos que esta solución es sólo parcialmente prefabricada, creándose una separación entre los hastiales y la clave - estas juntas aparecen también en el proyecto in situ.

En el plano social, en lo relativo a la seguridad e higiene en el trabajo, al prefabricar se evitan buena parte de los trabajos de obra sobre andamio, lo cual redunda en una probable disminución de la siniestralidad. Finalmente, y aunque en este caso es despreciable, no debe negarse un cierto interés social de la ejecución in situ, al considerar la creación de empleo en la zona del emplazamiento.

Respecto al medioambiente, al prefabricar se evitan vertidos de hormigón en obra, lo cual adquiere especial importancia al considerar el valor ecológico del emplazamiento; en una planta de prefabricados el volumen de hormigón sobrante se minimiza, y los restos - así como las aguas de lavado, etc. - se tratan con mayor pulcritud.

A partir de esta primera evaluación se plantea una cuantificación más detallada, a través de la construcción de las funciones de valor de los requerimientos afectados, recogidas en la
Tabla 3, donde $m_{x}$ y $M_{x}$ representan, respectivamente, los límites mínimo y máximo. Por otro lado, los valores que no tienen unidad adjunta se han evaluado mediante puntuación entre 1 y 10 ; en estos casos no existe un cálculo preciso, sino que debe asignarse un valor a cada alternativa que será resultado de la opinión de expertos. El parámetro $X_{3}$ se ha medido en la obra de fábrica OD 3, donde la necesidad de desagüe es mayor.

Tabla 3. Funciones de valor consideradas en la evaluación de la decisión

\begin{tabular}{|c|c|c|c|c|}
\hline & Funciones & $X_{i}$ & Indicador & Límites \\
\hline & Decrecientes & & C & $m_{x}=150.000 €$ \\
\hline $1(x)$ & & $x_{1}$ & Costes de las UD & $M_{x}=190.000 €$ \\
\hline & & & 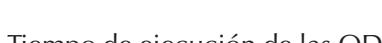 & $m_{x}=15$ días \\
\hline & $\mathbf{m}_{\mathbf{x}}$ & $\lambda_{2}$ & mempo de ejecucion de las UD & $M_{x}=40$ días \\
\hline & & $x$ & Vertidec en conctrucción & $m_{x}=0$ \\
\hline & & $\lambda_{3}$ & - & $M_{x}=10$ \\
\hline & Crecientes & $Y$ & Cauda do dosäت̈ & $m_{x}=600 \mathrm{~m}^{3} / \mathrm{s}$ \\
\hline$\left.v_{3}\left(x_{3}\right)\right]$ & & $x_{4}$ & Caudal de desague & $M_{x}=670 \mathrm{~m}^{3} / \mathrm{s}$ \\
\hline & & & Colidad do anobodo & $m_{x}=0$ \\
\hline & & $\lambda_{5}$ & Callada de acdodar & $M_{x}=10$ \\
\hline-1 & & $x$ & Grado de fisuración & $m_{x}=0$ \\
\hline & & $\lambda_{6}$ & & $M_{x}=10$ \\
\hline & & $Y$ & Soruridad do hos onorariog & $m_{x}=0$ \\
\hline & & $X_{7}$ & seguridad de ios operarios & $M_{x}=10$ \\
\hline
\end{tabular}

A partir de este despliegue del árbol se articula la medición según el tratamiento difuso explicado anteriormente. Las valoraciones de las estimaciones se presentan en la Tabla 4 (33). Hay que resaltar que en este caso, se ha estimado mediante formas rectangulares en vez de los trapecios dados en la Figura 2, por lo que se toman $\mathrm{a}=\mathrm{b}$ y $\mathrm{c}=\mathrm{d}$.

Tabla 4. Estimaciones relativas a los parámetros de respuesta

\begin{tabular}{|c|c|c|c|c|}
\hline Parámetros & Medida & Estimación & $\begin{array}{c}\text { Solución } \\
\text { prefabricada }\end{array}$ & $\begin{array}{l}\text { Solución } \\
\text { in situ }\end{array}$ \\
\hline \multirow{2}{*}{$\begin{array}{l}\text { X1: Coste de construcción } \\
\text { de las OD }\end{array}$} & \multirow{2}{*}{ Miles de euros } & $\mathrm{b}$ & 159.000 & 176.000 \\
\hline & & $\mathrm{C}$ & & 185.000 \\
\hline \multirow{2}{*}{$\begin{array}{l}\text { X2: Tiempo de ejecución } \\
\text { de las OD }\end{array}$} & \multirow{2}{*}{ Días } & $\mathrm{b}$ & 15 & 30 \\
\hline & & $\mathrm{C}$ & 18 & 35 \\
\hline \multirow{2}{*}{ X3: Caudal de desagüe } & \multirow{2}{*}{$\mathrm{m}^{3} / \mathrm{S}$} & $\mathrm{b}$ & 660,44 & 619,17 \\
\hline & & C & & \\
\hline \multirow{2}{*}{ X4: Calidad de acabado } & \multirow{8}{*}{$\begin{array}{l}\text { Escala cualitativa } \\
\text { del } 1 \text { al } 10\end{array}$} & $\mathrm{~b}$ & 7 & 5 \\
\hline & & C & 8 & 6 \\
\hline \multirow{2}{*}{ X5: Grado de fisuración } & & $\mathrm{b}$ & 1 & 3 \\
\hline & & $\mathrm{C}$ & 2 & 4 \\
\hline \multirow{2}{*}{$\begin{array}{l}\text { X6: Seguridad de los } \\
\text { operarios }\end{array}$} & & $\mathrm{b}$ & 7 & 5 \\
\hline & & $\mathrm{C}$ & 8 & 6 \\
\hline \multirow{2}{*}{$\mathrm{X} 7$ : Vertidos en obra } & & $\mathrm{b}$ & 8 & 5 \\
\hline & & C & 9 & 6 \\
\hline
\end{tabular}

Como consecuencia de la agregación y la obtención de las imágenes mediante la función $v(x)$ se obtiene el resultado para cada alternativa, según se recoge en la Figura 7. En este caso particular, al realizarse la estimación por intervalos, los trapecios genéricos a los que se aludía en la Figura 2 adoptan una forma rectangular.

A continuación se lleva a cabo la identificación, análisis y evaluación de riesgos. En la Tabla 5 se presentan los riesgos identificados y las medidas de respuesta, tanto 


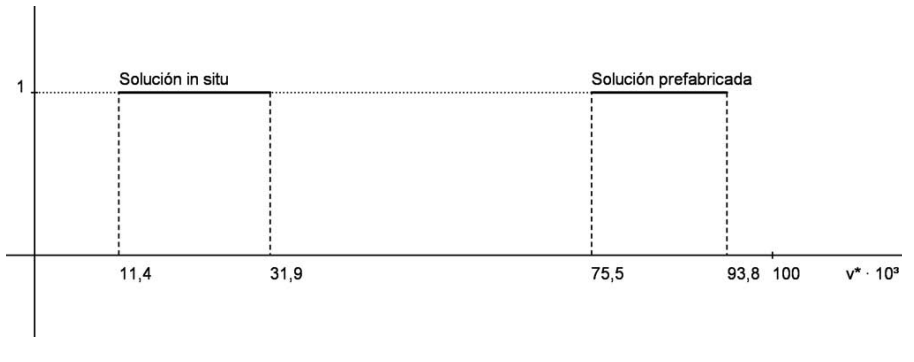

7

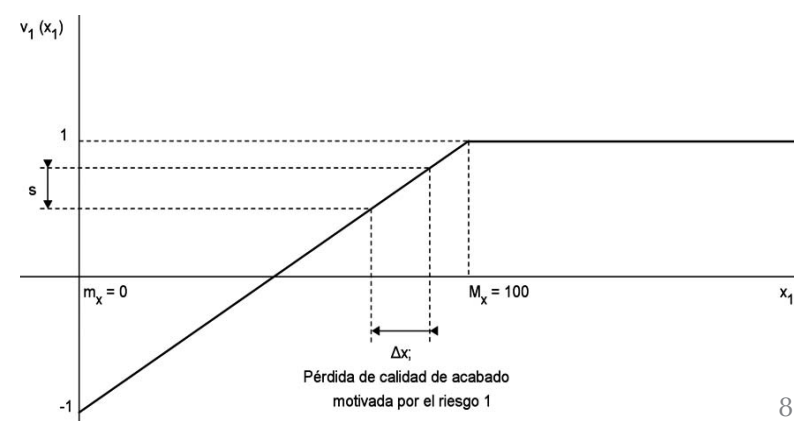

Tabla 5. Medición de los riegos considerados en la decisión en ambas alternativas

\begin{tabular}{|c|c|c|c|}
\hline Solución & Riesgo considerado & Medida & $\begin{array}{c}\text { Parámetros de } \\
\text { evaluación } \\
\text { de la seguridad }\left(x_{1}\right)\end{array}$ \\
\hline \multirow[b]{2}{*}{ Prefabricada } & $\begin{array}{l}\text { Filtración por incorrecto } \\
\text { sellado de las juntas }\end{array}$ & $\begin{array}{l}\text { Ninguna. Se considera } \\
\text { que no vale la } \\
\text { pena limpiarlo }\end{array}$ & $\begin{array}{l}\text { Estética de acabado } \\
\text { (regueros) }\end{array}$ \\
\hline & $\begin{array}{l}\text { Mal acabado por fallos } \\
\text { en el montaje } \\
\text { (resaltos entre piezas, } \\
\text { desconchados por golpes,etc.) } \\
\text { o defectos de fabricadión }\end{array}$ & $\begin{array}{l}\text { Exigir a la casa de } \\
\text { prefabricados } \\
\text { la reparación }\end{array}$ & $\begin{array}{l}\text { Estética de acabado } \\
\text { (aunque se repare } \\
\text { siempre se ve el } \\
\text { mortero o similar) }\end{array}$ \\
\hline \multirow[t]{3}{*}{ In situ } & $\begin{array}{l}\text { Mala ejecución por defectos } \\
\text { de encofrado, amasada } \\
\text { de hormigón } \\
\text { defectuosa, etc. }\end{array}$ & & Estética de acabado \\
\hline & Sobrecostes & & Aumentos de coste \\
\hline & Retrasos & & Aumentos de plazo \\
\hline
\end{tabular}

Tabla 6. Estimación de los riesgos considerados en la decisión

\begin{tabular}{|c|c|c|c|c|c|}
\hline & Riesgo & Probabilidad & Medición & Estimación & $\Delta \mathrm{x}$ \\
\hline \multirow{8}{*}{ Prefabricado } & \multirow{4}{*}{ R1: } & \multirow{4}{*}{0,2} & \multirow{4}{*}{$\begin{array}{l}\text { Pérdida de calidad } \\
\text { de acabado } \\
\text { (puntuación) }\end{array}$} & $\mathrm{a}$ & 2 \\
\hline & & & & $\mathrm{b}$ & 2 \\
\hline & & & & C & 3 \\
\hline & & & & d & 3 \\
\hline & \multirow{4}{*}{ R2: } & \multirow{4}{*}{0,3} & \multirow{4}{*}{$\begin{array}{c}\text { Pérdida de calidad } \\
\text { de acabado } \\
\text { (puntuación) }\end{array}$} & $\mathrm{a}$ & 1 \\
\hline & & & & b & 1 \\
\hline & & & & C & 2 \\
\hline & & & & d & 2 \\
\hline \multirow{11}{*}{ In situ } & \multirow{3}{*}{ R1: } & \multirow{3}{*}{0,2} & \multirow{3}{*}{$\begin{array}{c}\text { Pérdida de calidad } \\
\text { de acabado } \\
\text { (puntuación) }\end{array}$} & $\mathrm{a}$ & 3 \\
\hline & & & & b & 3 \\
\hline & & & & C & 4 \\
\hline & \multirow{4}{*}{ R2: } & \multirow{4}{*}{0,1} & \multirow{4}{*}{$\begin{array}{c}\text { Posible sobrecoste } \\
\text { de imprevistos } \\
\text { (euros) }\end{array}$} & $\frac{d}{a}$ & $\begin{array}{c}4 \\
3000\end{array}$ \\
\hline & & & & $\mathrm{b}$ & 3000 \\
\hline & & & & C & 6000 \\
\hline & & & & d & 6000 \\
\hline & \multirow{4}{*}{ R2: } & \multirow{4}{*}{0,1} & \multirow{4}{*}{$\begin{array}{l}\text { Posible retraso } \\
\text { de imprevistos } \\
\text { (días) }\end{array}$} & $\mathrm{a}$ & 10 \\
\hline & & & & b & 10 \\
\hline & & & & C & 15 \\
\hline & & & & $d$ & 15 \\
\hline
\end{tabular}

7. Resultado en términos difusos de la evaluación de alternativas sin considerar riesgos puros.

8. Visualización de la pérdida de valor producida por el efecto del riesgo. para el caso de la solución in situ como de la solución prefabricada. En dicha tabla se muestran también los parámetros considerados para el cálculo de la severidad una vez adoptadas las medidas de respuesta.

Estas pérdidas de calidad de acabado se miden a través de puntuación en una escala del 1 al 10. Para ello se utiliza una vez más el árbol de requerimientos, convenientemente desplegado. En este caso, al hacer referencia ambos al mismo parámetro, ya utilizado sin considerar los riesgos puros en el cálculo del valor, no es necesario repetir el proceso de despliegue e identificación de parámetros. Como se observa en la tabla anterior, no se ha considerado la influencia en el coste y el tiempo de construcción.
El resultado de la estimación de pérdida de calidad de acabado, para ambas soluciones se presenta en la Tabla 6 , donde $\Delta x$ es la posible pérdida producida por el riesgo medida en el parámetro $x$, puntuándose sobre una escala de 1 a 10.

Las probabilidades se calculan de forma subjetiva (no existen en esta caso datos que permitan otra alternativa para su estimación). Por otro lado, la severidad se evalúa según lo explicado en el apartado 2, de modo que, por ejemplo, el riesgo 1 supone una severidad correspondiente a la pérdida de valor que implica la peor calidad de acabado (Figura 8).

Desde el punto de vista del riesgo, el prefabricado aporta otras dos claras ventajas respecto a la ejecución in situ. En primer lugar, esta última presenta el problema de la carencia en el mercado de mano de obra especializada en el momento de ejecutar la obra. Ello implica que la impericia de los operarios pueda dar lugar a defectos en la ejecución que redundarán, como mínimo, en unas peores características estéticas. Sin embargo, en la industria de la prefabricación, la especialización y la optimización de rendimientos de trabajo son mayores, lo cual reduce el problema. Por otro lado, no existe incertidumbre sobre los valores del coste y del plazo. En cualquier caso, el coste de la parte prefabricada viene fijado por el contrato, mientras que el plazo no queda totalmente asegurado, pero sí cubierto por penalizaciones contractuales. De hecho, si el proveedor del prefabricado es suficientemente fiable esta incertidumbre puede considerarse despreciable. El resultado de la estimación de la posible pérdida de valor producida los estos riesgos considerados sobre la alternativa de ejecución in situ es la que se muestra en la Tabla 7.

A partir de estos resultados se calcula la pérdida de valor que supone la posible existencia de estos riesgos restando las citadas severidades al valor calculado inicialmente. El resultado es el recogido en la Tabla 7 y la Figura 9.

A continuación, siguiendo el flujo del proceso ACE cabría plantear la posible incompatibilidad de alguna de las alternativas consideradas con otros aspectos del proyecto. 
En este caso no se observa ninguna. Posteriormente se realiza un análisis de sensibilidad consistente en variar algunos de los valores de los pesos y de los parámetros para sopesar la variación que imprimen al resultado final del valor de las diversas alternativas. Este análisis señala que los tanteos realizados no introducen variaciones significativas en la evaluación realizada, es decir, que una pequeña modificación no produce cambios importantes en el resultado final.

Dado que no se han detectado incompatibilidades ni el análisis de resultados ha producido variaciones, los resultados finales corresponden a los recogidos en la Figura 9. Según se observa, el conjunto difuso asociado a la alternativa prefabricada presenta valores más elevados que los de la otra opción. Por tanto, a la vista de estos resultados se escoge la alternativa prefabricada por estimarse que aporta un mayor valor al conjunto del proyecto.

\section{CONCLUSIONES}

El estudio presentado se desenvuelve paralelamente en tres aspectos que confluyen finalmente hacia una conclusión, si bien cada uno de los aspectos tratados tiene validez desde su propio punto de vista:

I. El sistema integrado de decisiones (IDS), como herramienta de apoyo para la toma de decisiones, con base en la teoría de la lógica difusa se ha mostrado eficiente para la resolución del problema planteado. El entorno de uso de la herramienta es el de la gestión de proyectos de construcción. Integra múltiples aspectos (económico, temporal, funcional, social y medioambiental) analizando la satisfacción que aporta cada alternativa y evaluando los riesgos inherentes. El proceso de elección pasa por tres fases denominadas análisis, creatividad y evaluación.

II. El debate de la normalización del proceso de toma de decisiones para todo gestor de proyectos que tenga afán de excelencia profesional debe ser una referencia constante. Así, cuando existen evidencias
Tabla 7. Cálculo del valor integrado

\begin{tabular}{|l|c|c|c|c|c|c|c|c|c|c|}
\hline & \multicolumn{4}{|c|}{$\begin{array}{c}\text { Alternativa 1: } \\
\text { Solución prefabricada }\end{array}$} & \multicolumn{5}{c|}{$\begin{array}{c}\text { Alternativa 2: } \\
\text { Solución in situ }\end{array}$} \\
\cline { 2 - 13 } & $\mathrm{P}_{\mathrm{i}}$ & $\mathrm{a}$ & $\mathrm{b}$ & $\mathrm{c}$ & $\mathrm{d}$ & $\mathrm{P}_{\mathrm{q}}$ & $\mathrm{a}$ & $\mathrm{b}$ & $\mathrm{c}$ & $\mathrm{d}$ \\
\hline Valor sin riesgo $\left(\mathbf{v}^{*}\right)$ & 1 & & 0,0755 & 0,0938 & & 1 & & 0,0114 & 0,0319 & \\
\hline Severidad riesgo 1 (s1) & 0,2 & & 0,0016 & 0,0009 & & 0,2 & & 0,0021 & 0,0014 & \\
\hline Severidad riesgo 2 (s2) & 0,3 & & 0,0011 & 0,0004 & & 0,1 & & 0,0031 & 0,0015 & \\
\hline Severidad riesgo 3 (s3) & & & & & & & & 0,0018 & 0,0017 & \\
\hline Valor integrado & & & 0,0748 & 0,0935 & & & & 0,0103 & 0,0311 & \\
\hline
\end{tabular}

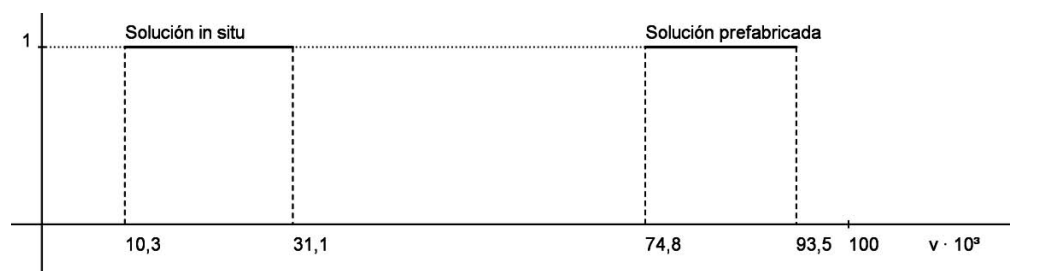

suficientes y recursos para ponderarlas, la búsqueda de la solución óptima no se puede obviar. Esta concienciación obliga a plantear con hondura la gestión de un proyecto desde su concepción.

III. Para la contrastación del método IDS se utiliza un caso sencillo (tres obras de drenaje en una urbanización) que ilustra pros y contras del prefabricado, resultando, en este caso, más favorable la solución prefabricada.

A pesar de ser los tres puntos anteriores aportaciones individuales de este trabajo, cabría extraer como conclusión colateral que el empleo de componentes prefabricados es en ocasiones la opción más sensata, y no sólo una buena práctica, en ingeniería y arquitectura. Y que esta decisión debe contemplarse desde el momento en que se planifica una obra. Queda identificada, además, la conveniencia de incluir en los planes de estudio de ingeniería y arquitectura nociones suficientes de prefabricación.

\section{AGRADECIMIENTOS}

Los autores de este artículo expresan su agradecimiento, especialmente, hacia las empresas que han facilitado la información necesaria para la realización de este trabajo: VERTIX, COPCISA y PREFABRICACIONES Y CONTRATAS.

\section{BIBLIOGRAFÍA}

(1) Pich-Aguilera, F., Batlle, T. y Casaldàliga, P.: "La arquitectura residencial como una realidad industrial. Tres ejemplos recientes", Informes de la Construcción, vol. 60, nº 512 (2008), pp. 47-60. doi: 10.3989/ic.08.040.

(2) Salas, J.: "De los sistemas de prefabricación cerrada a la industrialización sutil de la edificación: algunas claves del cambio tecnológico", Informes de la Construcción, vol. 60, nº 512 (2008), pp. 19-34. doi: 10.3989/ic.07.001.

(3) Sacks, R., Esatman, C. M. y Lee, G: "Process Model Perspectives on Management and Engineering Procedures in the Precast/Prestressed Concrete Industry", Journal of Construction Engineering and Management, vol. 130, $\mathrm{n}^{\circ} 2$ (2004), pp. 206-215. doi: 10.1061/(ASCE)0733-9364(2004)130:2(206).

(4) Jaillon, L. y Poon, C. S.: "Sustainable construction aspects of using prefabrication in dense urban environment: a Hong Kong case study", Construction Management and Economics, vol. 26, $\mathrm{n}^{\circ} 9$ (2008), pp. 953-966. doi: 10.1080/01446190802259043.

(5) Polat, G.: "Factors Affecting the Use of Precast Concrete Systems in the United States", Journal of Construction Engineering and Management, vol. 134, no 3 (2008), pp.169-178. doi: 10.1061/ (ASCE)0733-9364(2008)134:3(169). 
(6) Von Neumann, J. y Morgenstern, O.: Theory of Games and Economic Behavior, Princeton University Press, Princeton, 1947.

(7) Allais, M.: "Le Comportement de I'Homme Rationnel devant le Risque: Critique des Postulats et Axiomes de l'Ecole Americaine", Econometrica, vol. 21, no 4 (1953), pp. 503-546.

(8) Ellsberg, D.: "Classic and Current Notions of 'Measurable Utility'", The Economic Journal, vol. 64, $\mathrm{n}^{\mathrm{o}} 255$ (1954), pp. 528-556.

(9) Ellsberg, D.: "Risk, Ambiguity, and the Savage Axioms", The Quarterly Journal of Economics, vol. 75, no 4 (1961), pp. 643-669.

(10) Keeney, R. L. y Raiffa, H.: Decisions with Multiple Objectives: Preferences and Value Tradeoffs, Wiley, Hoboken, 1976.

(11) Simon, H. A.: "Rational Decision Making in Business Organizations", The American Economic Review, vol. 69, nº 4 (1979), pp. 493-513.

(12) Kahneman, D. y Tversky, A.: "Prospect Theory: An Analysis of Decision under Risk", Econometrica, vol. 47, nº 2 (1979), pp. $263-292$.

(13) Stone, P. A.: Building Econo my: Design, Production and Organisation: A Synoptic View, Pergamon Press, Oxford, 1966.

(14) Marcus, T. A., Hutton, G. H. y Devonald, A. D. G.: Value in Building, Applied Science Publishers, Londres, 1973, pp. 82-111.

(15) Hutton, G. H. y Devonald, A. D. G.: Value in Building. Applied Science Publishers, Londres, 1973, pp. 1-12.

(16) Burt, M.: A Survey of Quality and Value in Building. Building Research Establishment, Watford, 1978

(17) Elazouni, A. M. y Metwally, F. G.: "D-SUB: Decision Support System for Subcontracting Construction Works", Journal of Construction Engineering and Management, vol. 126, n 3 (2000), pp. 191-200. doi: 10.1061/(ASCE)CO.1943-7862.0000286

(18) Al-Subhi, K. M.: "Application of the AHP in project management", International Journal of Project Management, vol. 19, $\mathrm{n}^{\circ} 1$ (2001), pp. 19-27.

(19) Antuchevičiene, J., Zavadskas, E. K. y Zakarevičius, A.: "Multiple criteria construction management decisions considering relations between criteria", Technological and Economic Development of Economy, vol. 16, nº 1 (2010), pp. 109-125. doi: 10.3846/tede.2010.07.

(20) Wong, E., Norman, G. y Flanagan, R.: "A fuzzy stochastic technique for project selection", Construction Management and Economics, vol. 18, n 4 (2000), pp. 407-414.

(21) Rogers, M.: "Using Electre III to aid the choice of housing construction process within structural engineering", Construction Management and Economics, vol. 18, no 3 (2000), pp. 333-342.

(22) Koo, C-W. y otros: "A study on the development of a cost model based on the owner's decision making at the early stages of a construction project", International Journal of Strategic Property Management, vol. 14, nº 2 (2010), pp. 121-137. doi: 10.3846/ijspm.2010.10.

(23) Ormazábal, G., Viñolas, V. y Aguado, A.: "Enhancing Value in Crucial Decisions: Line 9 of the Barcelona Subway", Journal of Management in Engineering, vol. 24, $\mathrm{n}^{\circ} 4$ (2008), pp. 265-273. doi: 10.1061/(ASCE)0742-597X(2008)24:4(265).

(24) Villegas, N., De los Ríos, B. y Aguado, A.: "Value rate in highway cross-sections during its life cycle", International Review of Civil Engineering (IRECE), vol. 1, no 1 (2010), pp. 100-109.

(25) Aibinu, A. A.: Reducing Dispute in Construction Claims: The Role of the Decision-Making Process, 2009 AACE International Transactions.

(26) Yousefi, S., Hipel, K. W. y Hegazy, T.: "Attitude-based strategic negotiation for conflict management in construction projects", Project Management Journal, vol. 41, no 4 (2010), pp. 99-107. doi: 10.1002/pmj.20193.

(27) Heredia, R.: Dirección integrada de proyecto -DIP- 'project management', ETSII, Universidad Politécnica de Madrid, Madrid, 1995.

(28) Kerzner, H.: Project Management: A Systems Approach to Planning, Scheduling, and Controlling, Van Nostrand Reinhold, Nueva York, 2001.

(29) Serer, M.: Gestión integrada de proyectos, Edicions UPC, Barcelona, 2001.

(30) Chan, A. P. C., Chan, D. W. M. y Yeung, J. F. Y.: "Overview of the Application of 'Fuzzy Techniques' in Construction Management Research", Journal of Construction Engineering and Management, vol. 135, n 11 (2009), pp. 1241-1252. doi: 10.1061/(ASCE)CO.1943-7862.0000099.

(31) Kapliński, O. y Tamošaitiene, J.: "Game theory applications in construction engineering and management", Technological and Economic Development of Economy, vol. 16, nº 2 (2010), pp. 348-363. doi: 10.3846/tede.2010.22

(32) Aguado, A., Josa, A., Ormazábal, G., Estévez, B. y Cardim, A.: "Los requerimientos ambientales en la toma de decisiones sobre estructuras de hormigón", Hormigón y acero, no 234 (2004), pp. 63-73.

(33) Ormazábal, G.: EI IDS: Un nuevo sistema integrado de toma de decisiones para la gestión de projectos constructivos, Universitat Politècnica de Catalunya, Barcelona, 2002. Tesis doctoral.

(34) Šarka, V., y otros: "System of project multicriteria decision synthesis in construction", Technological and Economic Development of Economy, vol. 14, no 4, pp. 546-565. doi: 10.3846/1392-8619.2008.14.546-565.

(35) Mula, J., Poler, R. y García-Sabater, J. P.: "Capacity and material requirement planning modelling by comparing deterministic and fuzzy models", International Journal of Production Research, vol. 46, $\mathrm{n}^{\circ} 20$ (2008), pp. 5589-5606. doi: 10.1080/00207540701413912.

(36) Castro-Lacouture, D., y otros: "Construction Project Scheduling with Time, Cost, and Material Restrictions Using Fuzzy Mathematical Models and Critical Path Method", Journal of Construction Engineering and Management, vol. 135, nº 10 (2009), pp. 1096-1104. doi: 10.1061/(ASCE)0733-9364(2009)135:10(1096).

(37) Mills, G. R. y otros: "Applying a Universal Content and Structure of Values in Construction Management", Journal of Business Ethics, vol. 90, nº 4 (2009), pp. 473-501. doi: 10.1007/s10551-009-0055-7.

(38) Roca, P. y Aguado, A.: "Las filosofías asociadas a la construcción mediante elementos prefabricados de hormigón", Cemento-Hormigón, n 735 (1994), pp. 831-849.

(39) Aguado, A.; Del Caño , A.; De la Cruz, P.; Gómez, J.; Josa ,A.: "Sustainability assessment of concrete structures. The EHE approachRev", Journal of Construction Engineering and Management, ASCE, vol. 138, no 2 (2012), pp. 268-276. doi:10.1061/ (ASCE) CO.1943-7862.0000419.

(40) Saaty, T. L.: The Analytic Hierarchy Process: Planning, Priority Setting, Resource Allocation (Decision Making Series), McGraw-Hill, 1980.

(41) Sánchez, M., y otros: "Multiple-Criteria Evaluation for Value Management in Civil Engineering", Journal of Management in Engineering, vol. 21, no 3 (2005), pp. 131-137. doi: 10.1061/(ASCE)0742597X(2005)21:3(131). 